\title{
BMJ
}

\section{Effectiveness of rotavirus vaccination against childhood diarrhoea in El Salvador: case-control study}

\author{
Orbelina de Palma, epidemiologist, 'ilian Cruz, epidemiologist, ${ }^{1}$ Hector Ramos, epidemiologist, ${ }^{1}$ \\ Amada de Baires, microbiologist, ${ }^{1}$ Nora Villatoro, epidemiologist, ${ }^{1}$ Desiree Pastor, epidemiologist, ${ }^{2}$ \\ Lucia Helena de Oliveira, epidemiologist, ${ }^{3}$ Tara Kerin, biologist, ${ }^{4}$ Michael Bowen, research microbiologist, ${ }^{4}$ \\ Jon Gentsch, research microbiologist, ${ }^{4}$ Douglas H Esposito, epidemic intelligence service officer, ${ }^{4}$ \\ Umesh Parashar, epidemiologist, ${ }^{4}$ Jacqueline Tate, epidemiologist, ${ }^{4}$ Manish Patel, epidemiologist ${ }^{4}$
}

${ }^{1}$ Ministerio de Salud, San Salvador, El Salvador

${ }^{2}$ PanAmerican Health

Organization, San Salvador

${ }^{3}$ PanAmerican Health

Organization, Washington, DC,

USA

${ }^{4}$ Viral Gastroenteritis Team, Epidemiology Branch, DVD/NCIRD, MS-A47, Centers for Disease Control and Prevention, Atlanta, GA 30333, USA

Correspondence to: M Patel aul3@cdc.govAccepted: 16 March 2010

Cite this as: BMJ 2010:341:c2825 doi:10.1136/bmj.c2825

\section{ABSTRACT}

Objective To evaluate the effectiveness of a monovalent rotavirus vaccine against severe rotavirus disease and to assess its impact on diarrhoea in children aged less than 2 years after national introduction in El Salvador, a lowmiddle income country in Central America.

Design Matched case-control study.

Setting Seven hospitals in cities across El Salvador, January 2007 to June 2009.

Participants 323 children aged less than 2 years admitted with laboratory confirmed rotavirus diarrhoea and 969 healthy controls matched for age and neighbourhood. Main outcome measure Effectiveness of rotavirus vaccination ((1-adjusted odds ratio of vaccination) $\times 100$ ) against rotavirus diarrhoea requiring hospital admission. Results Cases and controls were similar for breast feeding, premature birth, maternal education, and socioeconomic variables. G1P[8] strains were identified in $92 \%$ of rotavirus cases. Effectiveness of two doses of vaccination against diarrhoea requiring hospital admission was $76 \%$ (95\% confidence interval 64\% to $84 \%)$. Protection was significantly lower $(\mathrm{P}=0.046)$ among children aged 12 months or more $(59 \%, 27 \%$ to $77 \%)$ compared with children aged 6-11 months (83\%, $68 \%$ to $91 \%$ ). One dose of vaccine was $51 \%$ ( $26 \%$ to $67 \%$ ) effective. At the sentinel hospitals, all admissions for diarrhoea among children under 5 declined by $40 \%$ in 2008 and by $51 \%$ in 2009 from the prevaccine year 2006 . Conclusions A monovalent rotavirus vaccine was highly effective against admissions for rotavirus diarrhoea in children aged less than 2 years in El Salvador and substantially reduced the number of such admissions in this low-middle income setting. The impact on disease epidemiology after vaccination, particularly among older children, warrants future attention.

\section{INTRODUCTION}

Globally, rotavirus related diarrhoea is responsible for 527000 deaths and 2.4 million admissions to hospital each year among children under 5 years. ${ }^{12}$ Two live attenuated oral rotavirus vaccines, a pentavalent bovine derived vaccine (RotaTeq; Merck Vaccines,
Whitehouse Station, NJ) and a monovalent human rotavirus vaccine (Rotarix; GlaxoSmithKline Biologicals, Rixensart, Belgium) with good efficacy against severe rotavirus infection and a reassuring safety profile could have a substantial impact on the global burden of severe diarrhoea in childhood. ${ }^{3-6}$ In 2006 the World Health Organization strongly recommended the inclusion of rotavirus vaccines into national immunisation programmes in American and European countries, and in April 2009 WHO extended this recommendation to all regions of the world. ${ }^{78}$

In making a global recommendation for rotavirus vaccines and noting the variable performance of the vaccines in different populations, WHO emphasised the need for further efficacy and effectiveness data, particularly in developing countries. ${ }^{8}$ For example, the efficacy of monovalent vaccine against severe rotavirus diarrhoea was 96\% (95\% confidence interval $83 \%$ to $99 \%$ ) in Europe, $83 \%$ (73\% to $90 \%$ ) in Latin America, $72 \%$ (40\% to 88\%) in South Africa, and 49\% $(11 \%$ to $72 \%)$ in Malawi. ${ }^{3-59}$ Similarly, the pentavalent vaccine prevented $98 \%(88 \%$ to $100 \%)$ of severe rotavirus infections in the United States and Finland, but effectiveness was $46 \%$ (18\% to 64\%) in Nicaragua, the only developing country setting for which data are available on the performance of the pentavalent vaccine. ${ }^{610}$ Experience with previous candidate rotavirus vaccines, as well as vaccines against polio, cholera, and typhoid, indicates that the immunogenicity and efficacy of live, oral vaccines can be impaired in developing countries of low and low-middle income. ${ }^{11-15}$ Therefore, studies on effectiveness after the introduction of vaccine and surveillance of rotavirus before and after the introduction of vaccine are vital to understand further the performance of rotavirus vaccines and their potential impact on the virus strains in target populations who are most likely to benefit from vaccination. ${ }^{16} 17$

El Salvador is a low-middle income country in Central America, with a gross national income of $\$ 2850$ (£1974; €2294) per capita and an annual birth cohort of around $158000 .{ }^{18}$ In October 2006 the ministry of 
health added the monovalent vaccine (recommending two doses) to its national childhood immunisation programme, providing the first opportunity to assess performance of the vaccine during programmatic use in a low-middle income country. The first dose is recommended at 2 months of age (between 6 and 14 weeks) and the second at 4 months of age (between 16 and 24 weeks); the maximum upper age limit is 24 weeks. Before the introduction of the vaccine, rotavirus was responsible for an estimated $35 \%$ of the admissions for diarrhoea in El Salvador, and although data on rotavirus strains from El Salvador are sparse, the G1P[8] strain predominated in 2001 and G2P [4] in 2006..$^{190}$ We assessed the effectiveness of two doses of the monovalent vaccine against rotavirus diarrhoea requiring hospital admission in El Salvador and compared the total number of admissions for diarrhoea and for rotavirus at sentinel hospitals in El Salvador before and after the introduction of the vaccine.

\section{METHODS}

From January 2007 to June 2009 we carried out a nationally representative matched case-control study to evaluate the effectiveness of the monovalent rotavirus vaccine against admission for rotavirus diarrhoea in children under 5 using a standardised WHO protocol. ${ }^{17} \mathrm{El}$ Salvador has 14 departments, five of which have the highest population density and constitute about $60 \%$ of El Salvador's annual birth cohort. From these regions the ministry selected seven hospitals where an estimated 48\% of El Salvador's annual admissions for diarrhoea occurred in children under 5 .

\section{Participants \\ Cases}

In January 2006 the ministry of health established a hospital based surveillance system to identify children with rotavirus diarrhoea who were admitted for dehydration at one of the seven sentinel hospitals. Active surveillance was carried out continuously in the emergency department and inpatient wards in children under 5 with acute diarrhoea, defined as three or more loose stools in a 24 hour period and with onset less than 14 days before the hospital visit. Healthcare providers in the seven hospitals were encouraged to notify the surveillance coordinator when treating a child under 5 with diarrhoea. The admission log was also reviewed daily to identify cases of diarrhoea. The surveillance coordinator interviewed the provider and the parent to assess case eligibility and subsequently collected bulk stool specimens within 48 hours of admission.

Specimens were stored at $2-8^{\circ} \mathrm{C}$ before daily transfer to the national laboratory, where rotavirus testing was done using a commercially available enzyme immunoassay (IDEIA; Oxoid, Ely, Cambridge). Specimens were frozen at $-70^{\circ} \mathrm{C}$ and shipped to the Centers for Disease Control and Prevention in Atlanta, where they were maintained at $-70^{\circ} \mathrm{C}$. Rotavirus positive specimens were then genotyped using previously described methods to determine the infecting strain. ${ }^{21-24}$

\section{Controls}

For each case we enrolled three controls from the neighbourhood who were individually matched to the case's date of birth (within 30 days). We selected controls through cumulative incidence sampling after the rotavirus season in 2007 and incidence density sampling on a weekly basis in 2008 and 2009. Controls were enrolled when interviewers visited homes to the left and right of the case's home until three controls were identified. Enrolment was restricted to one control per household. To avoid selection bias of positive respondents, we prohibited re-enrolment of controls once they were matched to a case. Written informed consent to participate was obtained from parents or guardians before enrolment.

\section{Variables}

During 2007, only information on vaccination status was gathered from cases and controls because of logistical delays from hiring and training study staff. During 2008-9, in addition to vaccination history, we obtained information on demographics, socioeconomic factors, birth weight, premature birth $(<36$ weeks' gestational age), current body mass index, history of breast feeding, and medical history; for cases, we also gathered information on clinical characteristics, treatment, and course of illness. We classified the gradient of severity among cases admitted for rotavirus diarrhoea using the previously described 20 point Vesikari scale. ${ }^{2526} \mathrm{We}$ computed effectiveness of the monovalent vaccine against rotavirus diarrhoea using severity scores of 11 or more and 15 or more.

\section{Data sources}

We interviewed the parents of cases and controls face to face. Vaccination was confirmed if the parent showed a vaccination card with the date of vaccination, type of vaccine used, and the child's name. If parents reported vaccination but did not possess a card, we reviewed vaccine records at the clinic where the child was reportedly vaccinated.

\section{Sample size for vaccine effectiveness}

We calculated precision based sample size estimates for a matched design under the following assumptions: vaccine coverage $50 \%$ and vaccine effectiveness $70 \%$, with a confidence limit width of $30 \% .{ }^{27}$ We estimated that about 150 cases of severe rotavirus diarrhoea (admission or Vesikari score $\geq 11$ ) and a control to case ratio of 3 to 1 would be sufficient for our primary analysis. Although we did not specifically power the study for subgroup analyses, we anticipated enrolling about 300 cases to allow us to carry out exploratory analysis on partial dose effectiveness and potential modification of effectiveness by age and to assess 
effectiveness against the most severe diarrhoea (Vesikari score $\geq 15$ ).

\section{Statistical analysis}

Our primary analysis assessed effectiveness of two doses of the monovalent vaccine against laboratory confirmed rotavirus diarrhoea requiring hospital admission. Consecutive cases admitted for laboratory confirmed rotavirus diarrhoea and born after 1 June 2006 were included in the vaccine effectiveness analysis, thus making them age eligible to receive the vaccine. Preplanned subgroup analyses were done to assess effectiveness by case severity, protection from one dose of vaccine, and duration of vaccine protection. We assessed partial series protection from one dose of vaccine and we also carried out an "intention to vaccinate" analysis to assess the effectiveness of one or more doses. To assess duration of protection we chose two specified groups for age at disease onset: 6-11 months and 12 months or more.

We considered cases and controls to be vaccinated if the most recent dose was administered 14 days before the case's hospital visit (reference date). We used conditional logistic regression to estimate the odds ratio of vaccination in cases compared with controls, assessing for effect modification by hospital, socioeconomic status, and age by including interaction terms in the model. To identify variables that changed the estimate

Table 1 Characteristics of rotavirus positive cases and neighbourhood controls in $\mathrm{El}$ Salvador, 2008 and 2009*. Values are numbers (percentages) unless stated otherwise

\begin{tabular}{|c|c|c|c|}
\hline Variables & $\begin{array}{c}\text { Rotavirus positive } \\
\text { cases } \dagger(n=251)\end{array}$ & $\begin{array}{l}\text { Neighbourhood } \\
\text { controls } \ddagger(n=753)\end{array}$ & $P$ value \\
\hline $\begin{array}{l}\text { Median (range) age } \\
\text { (months) }\end{array}$ & $10(1-24)$ & $10(1-25)$ & $0.96 \S$ \\
\hline Male & $161(64)$ & $378(50)$ & $<0.001$ \\
\hline History of breast feeding & $228(91)$ & $701(93)$ & 0.25 \\
\hline Daycare attendance & $9(4)$ & $17(2)$ & 0.94 \\
\hline Premature birth & $35(14)$ & $95(13)$ & 0.58 \\
\hline \multicolumn{4}{|l|}{ Maternal education: } \\
\hline None & $16(6)$ & $51(7)$ & \multirow{4}{*}{$0.17 \rrbracket$} \\
\hline Primary school & $162(65)$ & $528(70)$ & \\
\hline Secondary school & $52(21)$ & $136(18)$ & \\
\hline Tertiary school & $13(5)$ & $29(4)$ & \\
\hline $\begin{array}{l}\text { Median (range) No of } \\
\text { children in household }\end{array}$ & $1(0-10)$ & $1(0-21)$ & $0.006 \S$ \\
\hline $\begin{array}{l}\text { Median (range) No of } \\
\text { people in household }\end{array}$ & $2(0-10)$ & $2(0-9)$ & $0.04 \S$ \\
\hline \multicolumn{4}{|l|}{ Socioeconomic variables: } \\
\hline Electricity in home & $235(94)$ & 717 (95) & 0.19 \\
\hline $\begin{array}{l}\text { Owned motorised } \\
\text { vehicle }\end{array}$ & $24(10)$ & $71(9)$ & 0.96 \\
\hline Telephone in home & $178(71)$ & $529(70)$ & 0.82 \\
\hline Computer in home & $13(5)$ & $40(5)$ & 0.94 \\
\hline
\end{tabular}

*Variables not collected during 2007 surveillance year.

†Children admitted to hospital with acute diarrhoea who tested positive for rotavirus on enzyme immunoassay of stool.

$\ddagger$ Neighbourhood controls matched by age (within 30 days).

$\S$ Wilcoxon rank sum test.

ॠCochran-Armitage trend test.

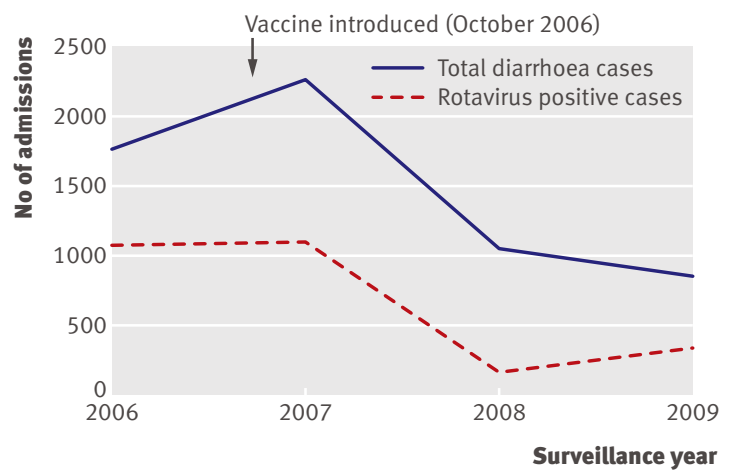

Diarrhoea and rotavirus related admissions among children aged less than 5 years at seven hospitals in El Salvador, January to June 2006-9

by more than $10 \%$ we used models controlling for factors potentially associated with vaccination and rotavirus disease - sex, history of breast feeding, daycare attendance, birth weight, and variables of socioeconomic status (maternal education, ownership of a motorised vehicle, and electricity, telephone, or computer in the home). These factors were selected on the basis of recommendations from a WHO guideline document. $^{17}$

We also compared the number of admissions to hospital for all cause diarrhoea and for rotavirus in 2008 and 2009 against the prevaccine year 2006, at the seven surveillance hospitals that applied the same surveillance methodology. We restricted this analysis to January to June, the months of peak rotavirus activity, or "rotavirus season."

We estimated the adjusted odds ratio using the exponential of the coefficient for the vaccination variable in the model. The 95\% confidence interval for the adjusted odds ratio was computed using the standard error of the coefficient ${ }^{28}$ and subsequently we calculated vaccine effectiveness for each group of controls as (1-adjusted odds ratio of vaccination) $\times 100 .{ }^{29} \mathrm{We}$ used the Wilcoxon rank sum test, trends test, or $\chi^{2}$ test to assess the differences in characteristics of children with rotavirus gastroenteritis and their matched controls. Statistical significance was designated as a two tailed $\mathrm{P}$ value $<0.05$. All analyses were done using SAS statistical software (version 9.2).

\section{RESULTS}

For the vaccine effectiveness study, 323 consecutive cases of rotavirus diarrhoea were age eligible for immunisation against rotavirus. Of the 81 children who were age eligible for vaccination during the 2007 rotavirus season (aged $\leq 6$ months), $72(89 \%)$ participated; the parents of the remaining children could not be located. Of the children who were age eligible for vaccination $(\mathrm{n}=256), 251(98 \%)$ participated: 113 during 2008 and 138 during 2009. All cases were admitted for dehydrating diarrhoea. Three neighbourhood controls matched by date of birth were identified for each case. Of the eligible controls who were at home during the community visit ( $\mathrm{n}=979), 969$ (99\%) 
Table 2 |Effectiveness of rotavirus vaccine against admissions for rotavirus related diarrhoea in El Salvador, 2007-9

\begin{tabular}{|c|c|c|c|c|}
\hline \multirow[b]{2}{*}{ Vaccine dose } & \multirow{2}{*}{$\begin{array}{l}\text { No }(\%) \text { of rotavirus } \\
\text { positive cases } \\
(n=323)\end{array}$} & \multicolumn{3}{|c|}{ Neighbourhood controls $(n=969)$} \\
\hline & & No (\%) & Odds ratio $(95 \% \mathrm{Cl})$ & $\begin{array}{l}\% \text { vaccine effectiveness } \\
(95 \% \mathrm{Cl})^{\star}\end{array}$ \\
\hline 0 dose (referent) & $99(31)$ & $153(16)$ & - & - \\
\hline 1 doset & $72(22)$ & $199(21)$ & $0.49(0.33$ to 0.74$)$ & 51 (26 to 67$)$ \\
\hline 2 dosesł & $152(47)$ & $617(64)$ & $0.24(0.16$ to 0.36$)$ & 76 (64 to 84$)$ \\
\hline
\end{tabular}

*Conditional logistic regression was used to calculate vaccine effectiveness. Models that included sex, history of breast feeding, daycare attendance, birth weight, and variables of socioeconomic status did not alter odds ratio and therefore final model included only vaccination as independent variable; adjustment factors were considered important if P 0.05 or estimates were changed by $10 \%$.

†Protection conferred after 14 or more days of vaccination (includes children admitted before additional doses). fProtection conferred after 14 or more days of vaccination.

participated in the study. The median age of cases and controls was 10 months (range 1-25 months). Cases were more likely than controls to be male $(64 \%$ v $50 \% ; \mathrm{P}<0.001)$ but were similar for breast feeding, premature birth, maternal education, and socioeconomic variables (table 1).

Cases were admitted to hospital for a median duration of two days; 98\% (247/251) received intravenous hydration and none died. Among the 239 cases with information on severity, $16(7 \%)$ had a Vesikari score of 10 or less, $223(93 \%) 11$ or more, and $54(22 \%) 15$ or more. Sufficient volume of stool was available for strain characterisation from $70 \%(225 / 323)$ of the cases. Among these samples, $92 \%(208 / 225)$ of the strains were G1P[8].

In cases $(\mathrm{n}=323)$ and controls $(\mathrm{n}=969)$, vaccine history was verified by vaccination card $(93 \%(n=301)$ cases, $97 \%(\mathrm{n}=944)$ controls $)$ or clinic record $(7 \%$ cases $(n=22), 3 \%(n=25)$ controls $)$. Adherence to the age recommendations for monovalent vaccine was good, with only $8 \%(14 / 175)$ of the vaccinated cases and $7 \%(48 / 687)$ of the vaccinated controls receiving their second dose of vaccine beyond 6 months of age. Among the cases, $31 \%(\mathrm{n}=99)$ were unvaccinated, $22 \%$ $(n=72)$ received one dose of the vaccine, and $47 \%$ $(\mathrm{n}=1522)$ received two doses (table 2). In contrast,

Table 3|Subgroup analysis to assess effectiveness of rotavirus vaccine against rotavirus diarrhoea of severity scores 11 or more and 15 or more, El Salvador 2008 and 2009*

\begin{tabular}{|c|c|c|c|}
\hline \multirow[b]{2}{*}{$\begin{array}{l}\text { Severity score and vaccine } \\
\text { dose }\end{array}$} & \multirow[b]{2}{*}{$\begin{array}{l}\text { No }(\%) \text { of rotavirus } \\
\text { positive cases }\end{array}$} & \multicolumn{2}{|c|}{ Neighbourhood controls } \\
\hline & & No (\%) & $\begin{array}{c}\% \text { vaccine effectiveness } \\
(95 \% \mathrm{Cl}) \dagger\end{array}$ \\
\hline Vesikari score $\geq 11$ : & $n=223$ & $n=669$ & \\
\hline 0 dose (referent) & $53(24)$ & 88 (13) & - \\
\hline 1 dose & $49(22)$ & $113(17)$ & $36(-9$ to 63$)$ \\
\hline 2 doses§ & $121(54)$ & $468(70)$ & 73 (56 to 84$)$ \\
\hline Vesikari score $\geq 15$ : & $n=54$ & $n=162$ & \\
\hline 0 dose (referent) & $15(28)$ & $22(14)$ & - \\
\hline 1 dosef & $14(26)$ & $26(16)$ & $45(-60$ to 81$)$ \\
\hline 2 doses§ & $25(46)$ & $114(70)$ & 83 (52 to 94$)$ \\
\hline
\end{tabular}

*Data on clinical variables for severity not available for $2007(n=72)$.

tFinal model included only vaccination as independent variable (see footnote to table 2); adjustment factors were considered important if $\mathrm{P}<0.05$ or estimates were changed by $10 \%$

‡Protection after one dose after 14 or more days of vaccination (includes children admitted before additional rotavirus vaccine doses).

§Protection after two doses after 14 or more days of vaccination.
$16 \%(\mathrm{n}=153)$ of community controls were unvaccinated, $21 \%(\mathrm{n}=199)$ received one dose of vaccine, and $64 \%(\mathrm{n}=617)$ received two doses (table 2). Vaccination rates did not differ by sex.

No confounding factors were identified in the study and thus the final model included vaccination as the independent variable only. Effectiveness against rotavirus diarrhoea requiring hospital admission after two doses of vaccine was $76 \%$ (95\% confidence interval $64 \%$ to $84 \%)$ and after one dose was $51 \%(26 \%$ to $67 \%$; table 2). Effectiveness estimates were similar between 2007 (77\%) and 2008-9 (75\%). In the intention to vaccinate analysis, protection from one or more doses of vaccine was $67 \%$ (95\% confidence interval $54 \%$ to $77 \%$ ). No heterogeneity in vaccine effectiveness was identified between hospitals $(\mathrm{P}=0.12)$ or between children of low and high socioeconomic status $(\mathrm{P}=0.89)$.

Subgroup analysis assessed effectiveness by severity and age. Two doses of vaccine were 83\% (95\% confidence interval $52 \%$ to $94 \%$ ) effective against rotavirus diarrhoea with a severity score of 15 or more and $73 \%$ (56\% to 84\%) effective against rotavirus diarrhoea with a severity score of 11 or more (table 3). We assessed duration of protection after vaccination by comparing rates of vaccination among cases and controls in children aged 6-11 months compared with those aged 12 months or older. Protection against admission for rotavirus related diarrhoea was 83\% (95\% confidence interval $68 \%$ to $91 \%$ ) in children aged 6 - 11 months and $59 \%(27 \%$ to $77 \%)$ in children aged 12 months or older (table $4 ; \mathrm{P}=0.046$ ).

\section{Vaccine impact}

Using the same surveillance methodology, between January 2006 and June 2009, 2702 of 8151 (33.1\%) children aged less than 5 years requiring overnight admission for episodes of diarrhoea at the seven surveillance hospitals were positive for rotavirus in their stool. Most (about 98\%) of the cases were identified during January to June. During these months the prevalence of rotavirus was $62 \%$ in $2006,49 \%$ in 2007 , $16 \%$ in 2008, and 40\% in 2009 (figure). Admissions for diarrhoea among children under 5 decreased from 1771 in 2006, before the introduction of vaccine, to 1066 in 2008 (40\% reduction) and to 863 in 2009 (51\% reduction). The number of laboratory confirmed rotavirus related admissions in children under 5 decreased from 1092 in 2006 to 171 in 2008 (84\% reduction) and to 342 in 2009 (69\% reduction) at the same surveillance sites (figure).

\section{DISCUSSION}

In April 2009, WHO strongly recommended the introduction of rotavirus vaccines into national immunisation programmes worldwide, but noted a need for ongoing monitoring of vaccine performance on the basis of variable efficacy of the vaccines in different populations. ${ }^{8}$ Under routine use in a low-middle income country, we have shown that the monovalent 
rotavirus vaccine provided $76 \%$ protection against admissions for rotavirus among children under 2 years, which was only slightly lower than the efficacy of $85 \%$ observed in a clinical trial of the monovalent vaccine in other middle income Latin American countries. ${ }^{34}$ Recent studies indicate that the population level benefits of vaccination are likely to be greater in the poorest settings with the highest incidence, and perhaps even greater than expected on the basis of direct effects of rotavirus vaccination. ${ }^{930}$ Moreover, the finding that one dose of monovalent vaccine confers about $50 \%$ protection indicates that vaccination could also have an impact between 2 and 6 months of age when a substantial proportion of admissions to hospital and deaths from rotavirus related disease occur. More encouragingly, routine use of monovalent vaccine has substantially reduced the health burden of admissions for rotavirus and all cause diarrhoea during the winter months in this setting. The $40 \%$ to $51 \%$ reduction in all cause diarrhoea among children under 5 was substantially greater than expected because few children aged more than 2 years were vaccinated during the study period, a potential indicator of indirect benefits to unvaccinated members of the community from interruption of transmission. Our finding of high effectiveness of the monovalent vaccine and noticeable impact over a three year study span in this challenging population reinforces the WHO recommendation for childhood rotavirus vaccination and provides further support for vaccination as an important measure in reducing global rotavirus related morbidity and mortality.

\section{Comparison with other studies}

The protection of $76 \%$ conferred by the monovalent vaccine in El Salvador seems to be greater than the

Table 4 | Effectiveness of rotavirus vaccine stratified by age at time of admission to hospital

\begin{tabular}{|c|c|c|c|}
\hline \multirow[b]{2}{*}{ Subgroups } & \multicolumn{2}{|c|}{$\begin{array}{c}\text { No/total No (\%) receiving two doses of vaccine } \\
\text { ( } v 0 \text { doses) }\end{array}$} & \multirow[b]{2}{*}{$\begin{array}{l}\% \text { vaccine effectiveness } \\
\qquad(95 \% \mathrm{Cl})\end{array}$} \\
\hline & $\begin{array}{l}\text { Rotavirus positive } \\
\text { cases }\end{array}$ & $\begin{array}{l}\text { Neighbourhood } \\
\text { controls }\end{array}$ & \\
\hline \multicolumn{4}{|l|}{$\begin{array}{l}\text { Admitted rotavirus } \\
\text { cases: }\end{array}$} \\
\hline All ages & $152 / 251(61)$ & $617 / 770(80)$ & 76 (64 to 84) \\
\hline Age 6-11 months & 49/63 (78) & $205 / 222(92)$ & 83 (68 to 91) \\
\hline Age $12-24$ months & 79/108 (73) & $284 / 335(85)$ & 59 (27 to 77$)$ \\
\hline \multicolumn{4}{|l|}{ Vesikari score $\geq 11 \dagger$ : } \\
\hline All ages & $121 / 174(70)$ & $468 / 556(84)$ & 73 (56 to 84$)$ \\
\hline Age 6-11 months & 45/57 (79) & $188 / 203$ (93) & 79 (47 to 91) \\
\hline Age $12-24$ months & 74/99 (75) & $263 / 309(85)$ & 58 (21 to 78$)$ \\
\hline \multicolumn{4}{|l|}{ Vesikari score $\geq 15 \dagger$ : } \\
\hline All ages & $25 / 40(63)$ & $114 / 136(84)$ & $83(52$ to 94$)$ \\
\hline Age 6-11 months & $11 / 16(69)$ & $52 / 56(93)$ & 92 (46 to 99) \\
\hline Age $12-24$ months & $14 / 19(74)$ & $58 / 68(85)$ & 58 (-67 to 89$)$ \\
\hline
\end{tabular}

Variables not collected for diarrhoea severity during 2007 surveillance year.

*Final model included only vaccination as independent variable (see footnote to table 2); adjustment factors were considered important if $\mathrm{P}<0.05$ or estimates were changed by $10 \%$.

†Protection conferred against rotavirus diarrhoea by two doses 14 or more days after vaccination. protection of about $50 \%$ conferred by the vaccine against rotavirus diarrhoea requiring admission in a similar study in the neighbouring country of Nicaragua, ${ }^{10}$ where the introduction of the pentavalent vaccine occurred simultaneously with the introduction of the monovalent vaccine in El Salvador. These differences may in part be attributable to differences in the severity of rotavirus disease among people evaluated in the two countries. Rotavirus vaccines have lower efficacy against milder disease, and 33\% of the cases with rotavirus admitted in Nicaragua compared with only $7 \%$ in El Salvador had a severity score of 10 or less. However, the effectiveness of the monovalent vaccine in El Salvador seemed to be $21 \%$ greater than that of the pentavalent vaccine in Nicaragua, even when the comparison was restricted to cases with the same severity. Differences in vaccine formulation and circulating strains during the study period might explain some of the variation, although both vaccines have provided good heterotypic and homotypic protection against common strains in high and middle income settings. $^{356}$ Differences in host and environment between the two populations could also be a factor, as El Salvador is a low-middle income country with gross national income of $\$ 2850$ per capita whereas Nicaragua is a low income country with a gross national income of $\$ 980$ per capita. The most impoverished settings may pose greater challenges (for example, higher inoculums of infection, greater rates of coenteric infections, gut enteropathy, micronutrient malnutrition, or high levels of circulating maternal antibodies) to the performance of oral rotavirus vaccines. ${ }^{1131}$ Indeed, the lowest observed efficacy of the monovalent vaccine, $50 \%$, has been in Malawi, one of the poorest settings in which the vaccine has been tested to date. Results of ongoing clinical trials of the pentavalent vaccine in poor countries of Africa and Asia are expected in the next few months and will provide key additional data to understand better the performance of oral rotavirus vaccines in challenging developing country settings.

Effectiveness of the monovalent vaccine seemed to be lower among children aged 12-24 months (59\%) compared with those aged 6-11 months (83\%), suggesting the possibility of waning immunity. This decline in protection in children aged more than 1 year was surprising, because overall protection against homotypic and many heterotypic strains was high and sustained (79-96\%) through two years in the European and Latin America monovalent vaccine trials. ${ }^{35}$ However, efficacy trials of previous rotavirus vaccines and studies of protection after natural rotavirus disease have also observed a decline in immunity to vaccine or wild virus, especially in poor settings. ${ }^{32-36}$ In Nicaragua, the pentavalent vaccine also showed a lower (but not statistically significant) effectiveness among children aged $12-19$ months. ${ }^{10}$ Similarly, in a small case-control study in an impoverished population in Brazil, the monovalent vaccine conferred good protection $(85 \%$, $95 \%$ confidence interval $54 \%$ to $90 \%$ ) during the first year against diarrhoea from fully heterotypic G2P[4] 


\section{WHAT IS ALREADY KNOWN ON THIS TOPIC}

The monovalent rotavirus vaccine has shown high efficacy and good safety under clinical trial settings in Europe and middle-high income countries of Latin America

A lower performance of live attenuated oral rotavirus vaccines has been observed in developing countries with the highest burden of severe rotavirus disease

\section{WHAT THIS STUDY ADDS}

A monovalent rotavirus vaccine was highly effective against admissions for rotavirus diarrhoea in children aged less than 2 years in El Salvador, providing $76 \%$ protection

The substantial decline in diarrhoea episodes and severe rotavirus disease in El Salvador indicates that the population level benefits of vaccination in this low-middle income setting may potentially be greater than expected on the basis of direct vaccine protection

The lower effectiveness among older children highlights the importance of monitoring disease epidemiology after vaccination through robust surveillance systems

strains, but declined significantly $(5 \%,-187 \%$ to $69 \%)$ among children older than 12 months. ${ }^{37}$ It is possible that waning of vaccine induced immunity is greater in developing countries where the short lived intestinal immune response to vaccination may predominate, and a systemic response may be less robust. ${ }^{31}$ Furthermore, immune responses against homotypic strains may be longer lasting than against heterotypic strains. ${ }^{33}$ Potential waning of immunity during the first 2 to 3 years of age could contribute to important changes in disease epidemiology, such as an increase in severe disease among older age groups compared with baseline, thus warranting close monitoring through surveillance and epidemiological studies. However, given the wide confidence limits, caution is warranted in interpreting findings of lower effectiveness among older children. Additional evaluations, including results from the second year of follow-up of ongoing clinical trials of the monovalent vaccine in developing countries in Africa, will help assess the duration of protection after vaccination.

\section{Strengths and limitations of the study}

The observational nature of case-control studies warrants discussion. We cannot be certain that controls in our study ideally represent the source population to which the cases belong. Matching the controls by age and neighbourhood is likely to lessen confounding bias from these factors. We were further reassured by similarities in measures of socioeconomic variables between cases and controls in our study. Although cases were more likely to be male, sex was not a confounder because vaccination rates were similar between the sexes. We were also able to confirm vaccination history by review of the vaccination card or registry on all cases and controls thus minimising potential misclassification of vaccination status. Misclassification of children who had been vaccinated to non-vaccinated is possible, in the event of a failure to document vaccination at time of receipt and could theoretically lower our effectiveness estimates. However, any such misclassification would likely be equal in both cases and controls and thus unlikely to affect our effectiveness estimates substantially. Logistical reasons prohibited us from blinding interviewers to case and control status, which might have introduced some bias. In addition, although cases and controls were matched on neighbourhood, in 2007 we collected data on rotavirus disease and vaccination status only. However, similar vaccine effectiveness estimates were observed when we restricted analysis to 2008-9 alone. It is also reassuring that during 2008 and 2009, cases and controls were similar for socioeconomic conditions, body mass index, birth weight, and breast feeding. All other surveillance methods were consistent during the four rotavirus seasons when rotavirus surveillance was done, and we do not suspect any substantial differences in healthcare seeking patterns between these years. Lastly, although an estimated $50 \%$ of the admissions for rotavirus in El Salvador occur at the study hospitals, these cases may not represent the full spectrum of severe cases in the population. Although clustering by hospital is possible, including hospital as a random effect in the conditional regression model did not substantially alter the presented findings.

With regard to the decline in all cause diarrhoea and rotavirus related admissions at the sentinel hospitals, secular variations in disease and changing reporting practices might have led to an observed reduction in disease after the introduction of vaccine. However, the contention that vaccine may have contributed to the reduction is supported by three findings: the high vaccine effectiveness in the case-control study, the substantial reduction in laboratory confirmed admissions to hospital for rotavirus at seven hospitals with active surveillance for diarrhoea before and after the vaccine was introduced, and the reduction in episodes of all cause diarrhoea observed during the winter season, when $98 \%$ of the laboratory confirmed rotavirus disease among children aged less than 5 years occurs in El Salvador. We were intrigued by the relative increase in rotavirus prevalence among children under 5 between $2008(16 \%)$ and $2009(40 \%)$. Although the short duration of surveillance limits our interpretation, the increase in rotavirus prevalence could be from secular variation in rotavirus and other causes of diarrhoea, or might reflect the decreased effectiveness among older children or an accumulation in 2009 of older susceptible children who were not exposed at an earlier age because of the noticeably diminished 2008 season. Despite an increase in rotavirus prevalence, a noticeable decline in the absolute number of admissions for rotavirus related diarrhoea and all cause diarrhoea in 2009 from 2006 still indicates a substantial impact from the vaccine. This finding highlights the need for good baseline surveillance for evaluating and interpreting post licensure changes in the epidemiology of rotavirus.

\section{Conclusions and policy implications}

In summary, the monovalent vaccine provided good protection against admissions for rotavirus diarrhoea in El Salvador. Protective immunity was obtained 
after one dose, which is administered at an age before an appreciable proportion of rotavirus cases occur. Carrying out rotavirus surveillance before and after vaccination and monitoring performance of rotavirus vaccination in different populations will allow a more complete assessment of the level and duration of protection in varied conditions and the potential influence of vaccination on disease epidemiology and virus strains.

We thank the sentinel hospital rotavirus surveillance team in El Salvador for enrolling participants.

Contributors: OdP, LC, HR, AdB, NV, and DP designed the study and collected the data. LHdO and UP designed the study, reviewed the data, and revised the manuscript. TK, MB, JG, JT, and DE analysed the data. MP oversaw the study and analysed the data. All authors reviewed the data, revised the manuscript, and have seen and approved the final version. The findings and conclusions in this report are those of the authors and do not necessarily represent the views of the Centers for Disease Control and Prevention.

Funding: This work was carried out under a collaborative arrangement with Program for Appropriate Technology in Health and was funded in part by the GAVI Alliance. The funders had no role in the study design, data collection and analysis, decision to publish, or preparation of the manuscript.

Competing interests: All authors have completed the unified competing interest form at www.icmje.org/coi_disclosure.pdf (available on request from the corresponding author) and declare (1) no financial support for the submitted work from anyone other than their employer; (2) no financial relationships with commercial entities that might have an interest in the submitted work; (3) no spouses, partners, or children with relationships with commercial entities that might have an interest in the submitted work; and (4) no non-financial interests that may be relevant to the submitted work.

Ethical approval: This study was approved by the PanAmerican Health Organization Program for Appropriate Technology in Health, and the El Salvador Ministry of Health.

Data sharing: No additional data available.

1 Parashar UD, Gibson CJ, Bresse JS, Glass RI. Rotavirus and severe childhood diarrhea. Emerg Infect Dis 2006;12:304-6.

2 Parashar UD, Burton A, Lanata C, Boschi-Pinto C, Shibuya K, Steele D, et al. Global mortality associated with rotavirus disease among children in 2004. J Infect Dis 2009;200(suppl 1):9-15S.

3 Linhares AC, Velazquez FR, Perez-Schael I, Saez-Llorens X, Abate H, Espinoza $F$, et al. Efficacy and safety of an oral live attenuated human rotavirus vaccine against rotavirus gastroenteritis during the first 2 years of life in Latin American infants: a randomised, double-blind, placebo-controlled phase III study. Lancet 2008;371:1181-9.

4 Ruiz-Palacios GM, Perez-Schael I, Velazquez FR, Abate H, Breuer T, Clemens SC, et al. Safety and efficacy of an attenuated vaccine against severe rotavirus gastroenteritis. $N$ Engl J Med 2006;354:11-22.

5 Vesikari T, Karvonen A, Prymula R, Schuster V, Tejedor JC, Cohen R, et al. Efficacy of human rotavirus vaccine against rotavirus gastroenteritis during the first 2 years of life in European infants: randomised, double-blind controlled study. Lancet 2007:370:1757-63.

6 Vesikari T, Matson DO, Dennehy P, Van Damme P, Santosham M, Rodriguez Z, et al. Safety and efficacy of a pentavalent human-bovine (WC3) reassortant rotavirus vaccine. N Engl J Med 2006;354:23-33.

7 World Health Organization. Rotavirus vaccines. Wkly Epidemiol Rec 2007;82:285-95.

8 Meeting of the immunization Strategic Advisory Group of Experts, April 2009-conclusions and recommendations. Wkly Epidemiol Rec 2009;84:220-36.

9 Madhi SA, Cunliffe NA, Steele D, Witte D, Kirsten M, Louw C, et al. Effect of human rotavirus vaccine on severe diarrhea in African infants. N Engl J Med 2010;362:289-98.

10 Patel M, Pedreira C, De Oliveira LH, Tate J, Orozco M, Mercado J, et al. Association between pentavalent rotavirus vaccine and severe rotavirus diarrhea among children in Nicaragua. JAMA 2009;301:2243-51.

11 Bresee IS, Parashar UD, Widdowson MA, Gentsch JR, Steele AD, Glass RI. Update on rotavirus vaccines. Pediatr Infect Dis J 2005;24:947-52.
12 Glass RI, Parashar UD, Bresee JS, Turcios R, Fischer TK, Widdowson MA, et al. Rotavirus vaccines: current prospects and future challenges. Lancet 2006;368:323-32.

13 John TJ. A developing country perspective on vaccine-associated paralytic poliomyelitis. Bull World Health Organ 2004;82:53-7.

14 Lagos R, Fasano A, Wasserman SS, Prado V, San Martin O, Abrego P, et al. Effect of small bowel bacterial overgrowth on the immunogenicity of single-dose live oral cholera vaccine CVD 103 HgR. J Infect Dis 1999;180:1709-12.

15 Levine MM. Enteric infections and the vaccines to counter them: future directions. Vaccine 2006;24:3865-73

16 Sanchez-Padilla E, Grais RF, Guerin PI, Steele AD, Burny ME, Luquero F. Burden of disease and circulating serotypes of rotavirus infection in sub-Saharan Africa: systematic review and metaanalysis. Lancet Infect Dis 2009;9:567-76.

17 WHO. Generic protocol for monitoring impact of rotavirus vaccination on gastroenteritis disease burden and viral strains. 2008. http:// whqlibdoc.who.int/hq/2008/WHO_IVB_08.16_eng.pdf.

18 UN Data. A world of information. 2010. http://data.un.org/.

19 Guardado JA, Clara WA, Turcios RM, Fuentes RA, Valencia D, Sandoval R, et al. Rotavirus in El Salvador: an outbreak, surveillance and estimates of disease burden, 2000-2002. Pediatr Infect Dis I 2004:23(suppl 10):156-60S

20 Patel MM, de Oliveira LH, Bispo AM, Gentsch J, Parashar UD. Rotavirus P[4]G2 in a vaccinated population, Brazil. Emerg Infect Dis 2008;14:863-5.

21 Das BK, Gentsch JR, Cicirello HG, Woods PA, Gupta A, Ramachandran M, et al. Characterization of rotavirus strains from newborns in New Delhi, India. / Clin Microbiol 1994;32:1820-2.

22 Gentsch JR, Glass RI, Woods P, Gouvea V, Gorziglia M, Flores J, et al. Identification of group A rotavirus gene 4 types by polymerase chain reaction. J Clin Microbiol 1992;30:1365-73.

23 Gouvea V, Glass RI, Woods P, Taniguchi K, Clark HF, Forrester B, et al. Polymerase chain reaction amplification and typing of rotavirus nucleic acid from stool specimens. J Clin Microbiol 1990;28:276-82.

24 Griffin DD, Nakagomi T, Hoshino Y, Nakagomi O, Kirkwood CD, Parashar UD, et al. Characterization of nontypeable rotavirus strains from the United States: identification of a new rotavirus reassortant (P2A[6],G12) and rare P3[9] strains related to bovine rotaviruses. Virology 2002;294:256-69.

25 Perez-Schael I, Guntinas MJ, Perez M, Pagone V, Rojas AM, Gonzalez R, et al. Efficacy of the rhesus rotavirus-based quadrivalent vaccine in infants and young children in Venezuela. N Engl / Med 1997;337:1181-7.

26 Ruuska T, Vesikari T. Rotavirus disease in Finnish children: use of numerical scores for clinical severity of diarrhoeal episodes. Scand I Infect Dis 1990;22:259-67.

27 Satten GA, Kupper LL. Sample size determination for pair-matched case-control studies where the goal is interval estimation of the odds ratio. J Clin Epidemiol 1990;43:55-9.

28 Hosmer DW, Lemeshow S. Applied logistic regression. 2nd ed. Wiley, 2000.

29 Orenstein WA, Bernier RH, Dondero TJ, Hinman AR, Marks JS, Bart KJ, et al. Field evaluation of vaccine efficacy. Bull World Health Organ 1985;63:1055-68.

30 Tate JE, Panozzo CA, Payne DC, Patel MM, Cortese MM, Fowlkes AL, et al. Decline and change in seasonality of US rotavirus activity after the introduction of rotavirus vaccine. Pediatrics 2009;124:465-71.

31 Patel M, Shane AL, Parashar UD, Jiang B, Gentsch IR, Glass RI. Oral rotavirus vaccines: how well will they work where they are needed most? J Infect Dis 2009;200(suppl 1):39-48S

32 Bernstein DI, Glass RI, Rodgers G, Davidson BL, Sack DA. Evaluation of rhesus rotavirus monovalent and tetravalent reassortant vaccines in US children. US Rotavirus Vaccine Efficacy Group. JAMA 1995;273:1191-6.

33 Chiba S, Yokoyama T, Nakata S, Morita Y, Urasawa T, Taniguchi K, et al. Protective effect of naturally acquired homotypic and heterotypic rotavirus antibodies. Lancet 1986;2:417-21.

34 Linhares AC, Gabbay YB, Mascarenhas ID, de Freitas RB, Oliveira CS, Bellesi N, et al. Immunogenicity, safety and efficacy of tetravalent rhesus-human, reassortant rotavirus vaccine in Belem, Brazil. Bull World Health Organ 1996;74:491-500.

35 Moulton LH, Staat MA, Santosham M, Ward RL. The protective effectiveness of natural rotavirus infection in an American Indian population. J Infect Dis 1998;178:1562-6.

36 Santosham M, Moulton LH, Reid R, Croll J, Weatherholt R, Ward R, et al. Efficacy and safety of high-dose rhesus-human reassortant rotavirus vaccine in Native American populations. J Pediatr 1997;131:632-8.

37 Correia JB, Patel MM, Nakagomi O, Montenegro FM, Germano EM, Correia NB, et al. Effectiveness of monovalent rotavirus vaccine (Rotarix) against severe diarrhea caused by serotypically unrelated G2P[4] strains in Brazil. / Infect Dis 2010;201:363-9.

Accepted: 16 March 2010 